\title{
REDES DE TRANSPORTE, ARTICULACIÓN TERRITORIAL Y DESARROLLO REGIONAL'1.
}

\section{TRANSPORT NETWORK, JOINT REGIONAL PLANNING AND DEVELOPMENT.}

\author{
Francisco Javier Antón Burgos \\ Universidad Complutense de Madrid \\ franjavi@ghis.ucm.es
}

Recibido: marzo, 2012.

Versión final aceptada: abril, 2013.

PALABRAS CLAVE: redes de transporte, accesibilidad, sistemas de transporte, redes de transporte, articulación territorial, desarrollo regional.

KEYWORDS: transport networks, accessibility, transportation systems, transportation networks, joint planning, regional development.

\section{RESUMEN}

La dinámica de las redes de transporte se muestra hoy como uno de los rasgos más significativos en relación con los cambios derivados de la globalización y las nuevas aplicaciones conceptuales y tecnológicas introducidas en la industria del transporte. La gestión de dichas redes y la implementación de modelos logísticos más eficientes, permiten la utilización de sus potencialidades derivadas en lo concerniente al diseño de políticas de articulación territorial en diferentes escenarios del mundo, así como su empleo como valiosos instrumentos al servicio del desarrollo regional en sus diferentes escalas espaciales.

\section{ABSTRACT}

The dynamics of transport networks shows today as one of the most significant features in relation to the changes brought about by globalization and new conceptual and technological applications introduced in the transportation industry. The management of such networks and the implementation of more efficient logistic models, allowing the use of their potentials derived with regard to joint design of territorial policies in different stages of the world, as well as their use as valuable tools in the service of regional development different spatial scales.

\footnotetext{
1 El presente artículo procede de la ponencia presentada en las Jornadas de Estudios Regionales: Redes y sus territorios: Incidencia en el desarrollo regional. AGE. Sevilla, Junio de 2012.
}

ISSN: 0212-8594 ISSN-e: 2340-2776. № DOI: http://dx.doi.org/10.12795/rea.2013.i30.02 


\section{LAS REDES DE TRANSPORTE EN UN MUNDO GLOBALIZADO.}

La malla mundial de redes de transporte se define como la suma de redes a diversas escalas (Global, continental, insular, nacional, regional, local...), que trasciende el marco de la mera contingencia de una ubicación concreta o de una movilidad particular tanto en personas como en mercancías. Dicho escenario ya fue atisbado por Olivier Dolfus en su obra Mondes Nouveaux, en la que las transformaciones que hoy se observan en el modelo mundial de los transportes ya estaba implícito en su análisis de movilidad con base en las actividades socioeconómicas, resaltando el dominio del sistema mundo ejercido desde los archipiélagos metropolitanos del hemisferio boreal.

Si se desagregan las mallas relativas a los ejes viarios de carreteras, ferrocarriles, grandes rutas marítimas y aéreas del mundo, se advierte la actualidad de lo ya descrito por Dolfus en 1996, con el aplastante predominio de los tejidos de malla originados en Europa Occidental y Estados Unidos, llamando también la atención los diferentes hiatos espaciales en los que ni se puede hablar de una verdadera malla estructurada y con actividad funcional: macizo septentrional de Sudamérica, Sahel, áreas vacías de Asia Central o Australia, etc.

Las mallas de carreteras son tanto más ostensibles y densas en Estados Unidos y Europa Occidental, del mismo modo que lo son las de carácter ferroviario, especialmente las localizadas en el centro-norte de la Unión Europea. En cuanto a las grandes rutas marítimas mundiales la modalidad de orígenes-destinos se concentra en tres grandes vectores de circulación: costa este de Norteamérica-Europa occidental, Asia oriental-costa occidental de Estados Unidos, y una circulación periférica que enlaza el sur de América, con el sur de África y su conexión por el Índico hacía Australia y Nueva Zelanda. Los puntos clave de dicha red se localización en las grandes áreas nodales de las costas oeste y este de Estados Unidos, los grandes puertos marítimos de Europa central-septentrional y los propios de los grandes gigantes asiáticos de Japón, China o Corea, junto con enclaves estratégicos de alto valor en las comunicaciones marítimas como Panamá, Suez, Mar negro o Singapur, que dominan a modo de atalaya los grandes circuitos comerciales por vía marítima tanto para el tráfico de combustibles como para el tráfico comercial de mercancías pesadas y grandes graneles.

La ruptura de los dispositivos rediales clásicos se produce con la expansión de las redes aéreas a nivel mundial, que en su configuración actual rompen con la tradicional dependencia derivada de la distancia y las localizaciones distales o de desenclave espacial, en las que las conexiones aéreas han modificado los mapas tradicionales de accesibilidad. De nuevo aparecen dos grandes localizaciones nodales al respecto, coincidentes con el centro y este de Estados Unidos y el área troncal más desarrollada de la Unión Europea, actuando como verdaderos elementos pivotales en torno a los cuales se articula la red mundial de enlaces aéreos, a los que habría que añadir un

ISSN: 0212-8594 ISSN-e: 2340-2776. № DOI:http://dx.doi.org/10.12795/rea.2013.i30.02 REA 30 (2013):27-47

http://www.publius.us.es/estudios_andaluces 
triángulo situado en Asia con vértices en Japón, China y Singapur, que indican los nuevos factores de polarización socioeconómica a nivel mundial ejercidos por los gigantes asiáticos emergentes.

La reducción de tiempo en el viaje, la ampliación de flotas aéreas y su capacidad de carga, tanto en pasaje como en mercancías, hacen que las lógicas tradicionales en el campo del transporte entren en quiebra por las posibilidades que ahora aporta el transporte aéreo en el contexto de la globalización, a lo que habría que añadir el reciente discurso de las "mobilities" (Cresswell,T., 2006; Urry,P., 2007; Knowles, R., Shaw, J. y Docherty, Y., 2008; Adey, P., 2009; Jensen, A., 2011).

Por ejemplo, Europa concentra el 23,2 \% del tráfico aéreo mundial de aeronaves, Estados Unidos el 35,5 \% (Destaca su tráfico interior y el gran número de aeronaves deportivas), Asía el 15, 9 \% (Peso específico de la carga aérea para grandes distancias), Centro y Sudamérica el $4,5 \%$, Pacífico un $2,6 \%$ y África un reducido $1,3 \%$, es decir, el discurso de modernidad aludido por Dolfus a finales del siglo XX pero con plena actualidad hoy día como soporte de una nueva estructuración de los grandes flujos de transporte en la escala mundial, en la que las nuevas oportunidades para el transporte personal jerarquizan las preferencias a la hora de elección del medio de transporte más efectivo para grandes contingentes, tal como ocurre con el transporte de pasajeros por vía aérea en el que Europa copa el 37,2 \%, Estados Unidos el 27,5\%, Asia el 9,3\%, Pacífico el $5 \%$, Centro y Sudamérica el 1,9\% y un exiguo 0,8 para África. (Todas las cifras señaladas proceden de IATA Air Traffic Statistics del año 2011).

\section{LA ACCESIBILIDAD ENTRE LOS GRANDES CENTROS NEURÁLGICOS DEL MUNDO.}

Salta a la vista que los factores con influencia en la accesibilidad espacial no son tan sólo de componente geográfico, económico o político, si no que devienen del mismo modo de otros aspectos tecnológicos y de la conformación técnica de las grandes redes mundiales de transportes, campo en el que la gestión integrada de las mismas se produce mediante las propuestas de un bloque de orientaciones y técnicas de gestión que cristaliza en el concepto de la logística, entendida de acuerdo con el Diccionario de la RAE como "el conjunto de medios y métodos necesarios para llevar a cabo la organización de una empresa, o de un servicio, especialmente de distribución", en cuyos comienzos destacó el papel de los medios militares para el transporte de efectivos y aprovisionamiento.

El nuevo cuadro de accesibilidad a escala global se puede visualizar particularizadamente a través de los grandes centros de producción, manufactura y consumo del mundo, así como en la distribución de los grandes archipiélagos metropolitanos en los que se concentra no sólo población, sino también poder político, capital, tecnología, empleo, elementos de la cultura preponderante, etc. (Garrison, G.

ISSN: 0212-8594 ISSN-e: 2340-2776. № DOI:http://dx.doi.org/10.12795/rea.2013.i30.02 
y Levinson, D., 2006). Norteamérica, costa oriental sudamericana, Europa occidental, central y septentrional, Decan y fachada oriental asiática, constituyen los actuales núcleos neurálgicos del presente sistema Mundo, aunque con las rápidas transformaciones que caracterizan la sociedad globalizada, nuevos enclaves podrán añadirse a futuro en dicha caracterización como parte del Golfo de Guinea, el Oriente Medio petrolero, Indonesia, etc.

\section{LOS SISTEMAS DE TRANSPORTE: INFRAESTRUCTURAS, REDES $Y$ GESTIÓN.}

Cinco son los apartados que en la actualidad nos permiten caracterizar los sistemas de transporte: infraestructuras, nodos de transporte o "hubs", redes de transporte, balance energético y gestión de los mismos.

Las infraestructuras son ahora un elemento más de los sistemas de transporte, imprescindibles si, pero no objeto exclusivo y único del desenvolvimiento de dichos sistemas (Levinson, D., 2011). La perspectiva clásica y tradicional del análisis topológico o pormenorizado de nodos concretos, aún de innegable interés, ha dado paso a la valoración de otros tantos elementos que condicionan la actividad a desarrollar sobre las infraestructuras mismas, en las que la toma de decisiones ya políticas o meramente técnicas, suponen en la práctica una forma determinada de entender la gestión de sistemas de transporte específicos o generales.

La infraestructura no tiene ya sentido en sí misma, sino como elemento que coadyuba a la articulación de sistemas complejos más amplios, tanto en dimensión como en intensidad de tráfico o especificad en el campo del transporte (Logística global o integrada, técnicas de "hubing", sistemas "puerta a puerta", "supply chain", etc.). Las infraestructuras individualizadas no tienen hoy el menor sentido en el complejo mundo del transporte, en el que todo funciona "en red", es decir asumiendo cada nodo o enclave un papel determinado dentro de una jerarquización vertical y horizontal - incardinada a su vez - en conjuntos holísticos sistémicos basados en la interdependencia y coalescencia de aportación. Conceptos como "renta de localización", "factor de especialización" o "disponibilidad de recursos", se presentan hoy como rasgos de especial singularidad complementarios en muchos casos, en otros como elementos determinantes, a la hora del diseño de la arquitectura de los propios sistemas de transporte (De Dios Ortuzar, J. y Willumsen, G., 2001). Modelos como los derivados de la Política Común de Infraestructuras de Transporte de la Unión Europea son emblemáticos al respecto, poniendo de manifiesto las aseveraciones hechas, es decir, una realidad cada vez más compleja en la que intervienen con un papel determinante los objetivos finalistas que se quieren alcanzar derivados de la influencia de planteamientos políticos, ideario económico o filosofía técnica de diseño. 
Tres componentes destacan con esta consideración en el análisis de las infraestructuras de transporte: características físicas de las mismas, su papel como elemento al servicio de la comunicación (medido con variables económicas, sociales y ambientales) y fórmulas de gestión más adecuadas según los objetivos definidos con anterioridad.

Buena parte de la definición del diseño de las redes de transporte reposa en la determinación del tipo de nodo o "hub" a construir o recrear, entendido como centro activo de transporte (Instalaciones, oferta de servicios, capacidad de almacenaje, sistemas de aproximación al nodo, componente ambiental, etc.), dotado de un cierto grado de polarización sobre el sistema en el que se inserta y con capacidad de afectar al entorno espacial en el que se ubica espacialmente (Dooms, M. et al., 2007).

La gestión óptima de hubs asentados, la elección de puntos para la localización de nuevos hubs y la reorganización de nodos para dar acogida a distintas funciones en un esquema jerarquizado, son posiblemente algunos de los tópicos más extendidos en el campo de la tecnología del diseño nodal, en la que confluyen presiones e intereses variados procedentes de los distintos tipos de administración escalar pública, agrupaciones empresariales y usuarios.

El papel social que puede derivarse de la existencia o actividad de un hub es igualmente muy tenida en cuenta a la hora del diseño de tejido territorial, máxime en tiempos de profunda crisis estructural en los que corresponde estudiar las posibilidades de generación de nuevas sinergias y expectativas (Actividad económica, creación de renta, captación de actividades, empleo, innovación y nuevas oportunidades, etc.), o la implementación de nuevos modelos territoriales en los que parece obligada la "recreación" de anteriores tejidos productivos, urbanos o de objetivos sociales, dentro de la óptica de la "competitividad territorial" o bien de la "complementariedad espacial" enunciada desde hace años por autores como Boisier.

Las redes de transporte presentan una arquitectura variable y redimensionable en el tiempo y en la forma, del mismo modo que ocurre con los complejos regionales específicos y espaciales en general a los que dan servicio. De hecho en multitud de ocasiones la trama de muchas redes de transporte ha sido el soporte efectivo de ciertos modelos de desarrollo, colaborando en la transformación en positivo de un territorio que había quedado obsoleto en cuanto a planteamientos de accesibilidad, capacidad de tráfico, movilidad interior, etc., tal como puede observarse en numerosos casos asociados a una dinámica de crecimiento en áreas turísticas, en contextos urbanos o en la expansión de nuevas áreas metropolitanas, transfronterizas o ámbitos litorales.

Los argumentos para aprovechar las sinergias de todo tipo que generan las redes de transporte, se fundamentan en la formulación de distintos tipos de redes atendiendo a diferentes necesidades del territorio en el que se sitúan, favoreciendo así su inserción en circuitos intermodales monográficos o de envergadura regional, nacional o incluso

ISSN: 0212-8594 ISSN-e: 2340-2776. № DOI:http://dx.doi.org/10.12795/rea.2013.i30.02 
global. El cualquier caso, la dimensión local de la presencia de redes de transporte exige una perspectiva de detalle para abordar sus particularidades, tanto como para el perfil de los objetivos a conseguir con su actividad.

Sin entrar en un mayor nivel de detalle, puede hablarse de diferentes tipos de red en función de las características del servicio que deben cumplir y de las actividades económicas y/o territoriales que deben soportar. La cuestión escalar o de dimensionamiento de la red, obedecen en multitud de casos a particularidades propias del territorio en el que se insertan, de modo que no puede hablarse de un taxón único a emplear, ya que además existe en los últimos tiempos una tendencia muy acentuada en cuanto al empleo de distintos modelos de red en un mismo espacio (económico, social, cultural, servicios...) o territorio físico.

Los "sistemas en red" corresponden con el más alto grado de desarrollo de una red de transporte, por lo común monográfica (aérea, marítima, logística de mercancías, correos, etc.), basada en una articulación jerárquica de sus nodos para garantizar con eficacia la direccionalidad de sus flujos (flotas, pasajeros o mercancías), y garantizar del mismo modo la velocidad de movimiento de los mismos, dentro de unos parámetros de funcionamiento abarcables de acuerdo con la capacidad de gestión de tráfico de la propia red. Los sistemas en red permiten a su vez la interoperatividad con otras redes en paralelo, como por ejemplo mallas urbanas con redes metropolitanas (metro ligero, transporte suburbano, cercanías ferroviarias...), nodos intermedios de conexión y distribuidores (sistemas "by pass") o tráfico minorista.

Los sistemas "hub and spoke" o de aporte y dispersión suponen uno de los modus operandi más extendido dentro del mundo del transporte, en los que desde nodos de rango inferior (feeders) se aportan flujos de tráfico hacía nodos dominantes a elección del operador, desde los cuales se encaminan flujos sumatorios de los recibidos hacia otros nodos dominantes de su propia red o de otras redes paralelas u operadores del mismo negocio (Elhedeli, S. y Hu, F., 2005) Desde ellos se producirá un fenómeno de dispersión hacia otros nodos menores, contribuyendo a la distribución de tráfico a puntos de más baja jerarquía o entidad dentro de diferentes redes. Esta es la modalidad de transporte aéreo más utilizada dentro de las grandes compañías aéreas y grandes operadores de logística de mercancías en el mundo, para lo cual necesitan asegurar el origen de su actividad mediante la captación de nodos de rango menor (secundarios o de tercer nivel), como elemento de captación de tráfico y como base operativa del modelo.

Muy conocida en Europa, y en particular en España, es la forma de seleccionar los hubs de tráfico por parte de las compañías aéreas de bajo coste o "low cost", que buscan aeropuertos de rango secundario, "slots" o frecuencias horarias de operación vacías o de menor coste, con destinos en aeropuertos principales a horarios de menor demanda. Todo ello sumado a la obtención de ayudas económicas de la administración

ISSN: 0212-8594 ISSN-e: 2340-2776. № DOI:http://dx.doi.org/10.12795/rea.2013.i30.02 REA 30 (2013):27-47

http://www.publius.us.es/estudios_andaluces 
local o regional, al objeto de asegurar regularmente dichos servicios a ciudades medias y pequeñas comunidades.

El sistema hub and spoke es igualmente practicado por los grandes operadores de logística de transporte (carretera, mixto, entregas urgentes, etc.), que concentran en puntos bien comunicados y con accesibilidad a grandes centros de almacenamiento y distribución, realizando parte del encaminamiento mediante rutas de horario nocturno, cuidando mucho sus relaciones con distribuidores minoristas asociados, consiguiendo así unas altas tasas de eficiencia (Yerra, B. y Levinson, D., 2005).

El sistema "Foliated Transportation System" (FTS), es aquel que está basado en la utilización de prácticas propias de los dos anteriores descritos, procurando obtener las sinergias propias de ambos, aplicando además un uso intensivo de la práctica de la intermodalidad de modos de transporte, favoreciendo de ese modo la concentración de volúmenes para baratar costes y obtener múltiples canales de encaminamiento de la carga (Hakimian, H. y Zandi, M., 2009) Por ello los FTS presentan una fuerte interacción con los operadores minoristas de distribución y captación, sin los cuales no podrían asegurar la concreción de su negocio.

Las redes "Point to Point Network" (PPN) suponen un cuarto sistema de transporte especializado en objetos con poco peso, volumen pequeño y alto valor añadido como correo rápido, transporte de documentos, transporte de objetos valiosos o de carácter perecedero como tráfico bancario o prensa. En todas sus modalidades el coste es alto y se garantizan las horquillas horarias de recogida y entrega a domicilio o en pequeñas centrales de ubicación céntrica, hecho que facilita mucho su demanda por parte de profesionales, en procesos legales o judiciales y por empresas especializadas.

Completando el espectro de de redes de transporte cabe reseñar la existencia de redes de estricto ámbito regional y mini-redes (muchas de ellas operando en (ámbitos urbanos). Las primeras cuentan con un cierto grado de especialización en el entorno en el que operan, conociendo bien las posibilidades de transporte a esa escala y contexto espacial, siendo frecuentes las alianzas operativas con otros agentes de regiones colindantes, mientras que las mini-redes utilizan pequeñas empresas distribuidoras de detalle tanto para productos de entrega urgente, perecederos o vinculados al e-commerce (grandes almacenes, seguros, servicios en general $y$ televenta (Andrea, D., Ciolfi, A. y Gray, B., 2011).

El balance energético de un sistema de transporte no se concibe hoy en día como el mero balance del gasto en combustible y energía que consume un conjunto de infraestructuras y flotas, sino más bien un todo ordenado en el que se valoran los recursos puramente energéticos necesarios para la operación del sistema, los recursos materiales tangibles (flotas, recursos humanos, instalaciones, equipamientos, financiación, canales de venta...) e intangibles (imagen de marca, publicitación, prestigio empresarial...), tan imprescindibles para poder competir en un mercado global. De modo que dicho balance energético se abre en tantos cuantos capítulos que 
precisan una valoración de imputs-outputs, cara a valorar la sostenibilidad del modelo explotado y sus posibilidades futuras de pervivencia en escenarios caracterizados tantas veces por su mutabilidad o volatilidad.

La gestión se muestra como una de las piedras angulares en el desenvolvimiento de los sistemas de transporte, fundamentados ahora no exclusivamente en la operación monográfica de una infraestructura, red o flota, sino en todo un contexto político, económico y social, en el que la eficacia del sistema depende de numerosos criterios y decisiones empresariales (De Brucker, K., Macharis, C., y Verbeke, A. 2011). Los planteamientos para el management de los sistemas de transporte responden a menudo en posicionamientos multicriterio (Dooms, M., 2010), basados en planes de actuación a corto y medio plazo, de los cuales los primeros son los verdaderamente más comprometidos, al implicar estratégicas de mercado y tácticas inmediatas de arriesgado alcance o éxito discutible, tal como ocurre con la valoración de objetivos y costes energéticos de cada sistema de transporte, sustitución de flotas o modernización de instalaciones.

\section{ALGUNAS RESPUESTAS DE LA INDUSTRIA DEL TRANSPORTE.}

Grandes operadores de transporte de rango mundial se están planteando a nivel formal la situación presente y futura de sus escenarios económicos y sociales, proponiendo determinadas alternativas de funcionamiento, cooperación y comportamiento ambiental para favorecer un futuro ordenado y sostenible tanto de sus actividades como del contexto global en el que se desenvuelven.

El caso del gigante de las comunicaciones marítimas Maersk, en su nueva hoja de ruta "A new roadmap for a more sustainable world" de 2012, plantea una serie de premisas para su actuación como que el mundo se encuentra en un profundo e intenso proceso de cambios, que la sostenibilidad es provechosa, cómo realizar la cadena de distribución, cómo optimizarla reduciendo las emisiones de $\mathrm{CO}^{2}$ e incrementar el rendimiento de la cadena de distribución. Como alternativa procedimental marca varios objetivos a alcanzar: reducir las emisiones de $\mathrm{CO}^{2}$ al $50 \%$ en la flota de barcos tipo triple-E, incentivar la eficiencia de los beneficios y se plantea si a futuro el combustible a emplear será el biofuel sustituyendo a los derivados de los hidrocarburos (Figura 1).

A título orientativo los planteamientos programáticos enunciados pueden considerarse como un referente para el sector, tanto como ideario a emular, como para la mejora de buenas prácticas en el orden ambiental y de la seguridad en el tráfico marítimo que preconiza.

Otro ejemplo de trascendencia global en el mundo del transporte es la aparición de las llamadas "alianzas aéreas mundiales", consolidadas ya desde comienzos del presente

ISSN: 0212-8594 ISSN-e: 2340-2776. № DOI:http://dx.doi.org/10.12795/rea.2013.i30.02 
siglo al objeto de compartir beneficios entre las aerolíneas coaligadas en dichas alianzas. Dichos beneficios se centran en una ampliación y optimización de la red de vuelos compartidos, reducción de costes, en inversiones y flotas, más amplia oferta de vuelos, incremento de los destinos, menores tiempos de viaje y precios más competitivos para el pasajero.

En suma, una fórmula de concentración de medios y efectivos en pos de una mayor rentabilidad y sostenibilidad futura del sector aéreo, en momentos de fuerte encarecimiento del precio del combustible y crisis sectorial focalizada en muchos países, pero con la esperanza de mejores horizontes futuros como consecuencia de los procesos de liberalización sectorial y "cielos abiertos" (Figura 2).

Figura. 1. Maersk, nueva hoja de ruta para un mundo más sostenible.

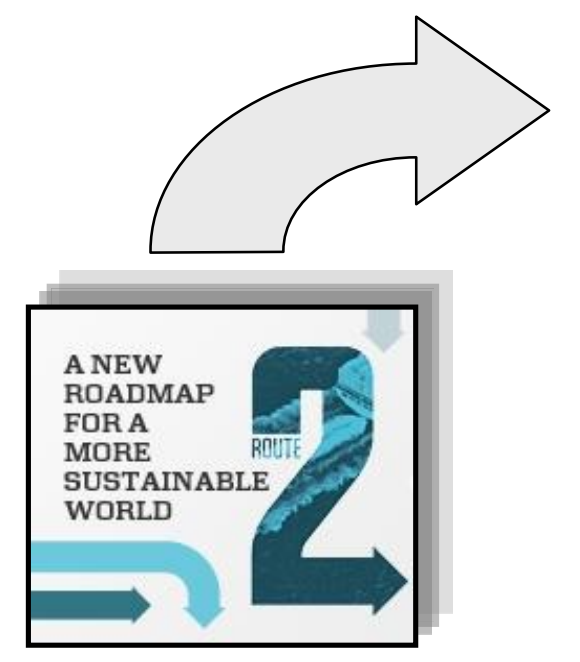

n WORLD IS CHANGING

- SUSTAINABILITY IS PROFITABLE

- WHAT ABOUT SUPLY CHAIN WASTE ?

- CUTTING CUPPLY CHAIN WASTE

(Higher profit and less $\mathrm{CO}^{2}$ )

- INCREASING SUPPLY CHAIN VALUE

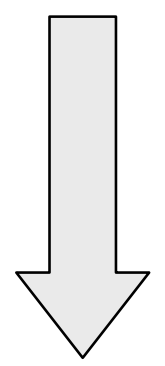

THE FUTURE OF TRANSPORTATION:

$\triangle$ THE TRIPLE-E CLASS REDUCES THE CO ${ }^{2}$ EMISSIONS BY $50 \%$

$\triangle$ COST EFFICIENT RECICLING

$\triangle$ IS BIOFUEL THE WAY FORWARD?

Fuente: Maersk, 2012.

En el año de referencia de 2001, ya que después se han producido diferentes cambios en la composición de las alianzas aéreas, las cuatro existentes en tal año consiguieron

ISSN: 0212-8594 ISSN-e: 2340-2776. № DOI:http://dx.doi.org/10.12795/rea.2013.i30.02 REA 30 (2013):27-47

http://www.publius.us.es/estudios_andaluces 
obtener el 62,4 \% de cuota del mercado mundial, sumando un total de 832.000 empleados con alto nivel de cualificación y una flota con varias decenas de miles de aviones, lo que indica con claridad la trascendencia y resultados de este proceso de alianzas.

Una tercera respuesta a valorar, aún a título indicativo y sin entrar en casos puntuales, es la articulación de redes regionales dentro del estado autonómico español, en el cual diferentes comunidades tienen transferidas las competencias en materia de transporte regional. Los objetivos a fecha de hoy puede decirse que han sido y son muy variados, casos en los que ha primado la construcción y reconstrucción de redes, frente a muy pocos procesos de "deconstrucción" impulsados por lo gobiernos autonómicos junto a algunas actuaciones puntuales de innovación.

Figura 2. Respuesta a la globalización: alianzas aéreas mundiales en 2001.

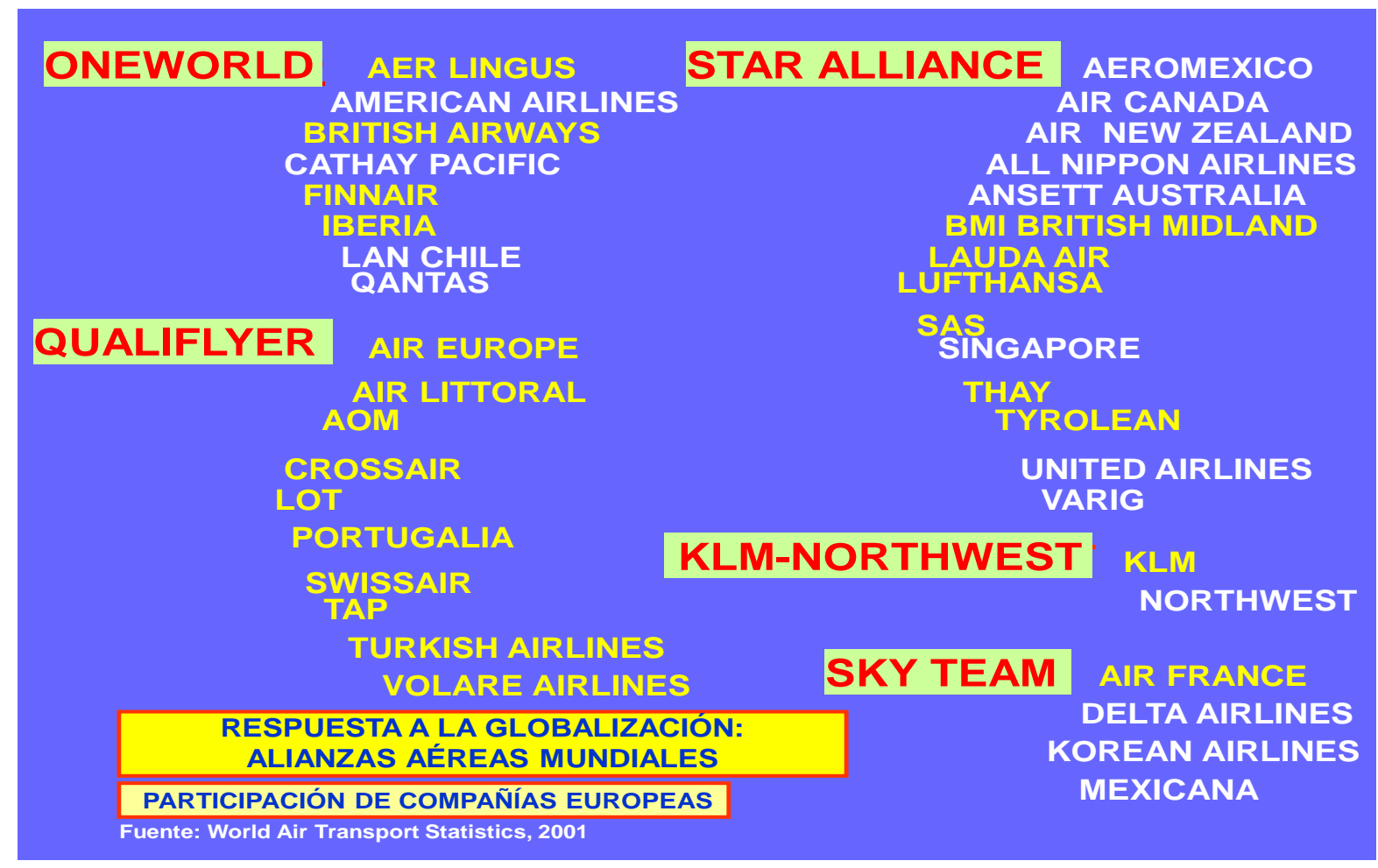

Fuente: Elaboración propia a partir de datos de World Air Transport Statistics, 2001.

En cuanto a la evolución de las infraestructuras en el marco regional, se aprecian varios componentes a destacar: en el campo puramente físico se han implementado diferentes actuaciones constructivas, se incide en la calidad de las mismas y se mejoran comparativamente ratios de unidades construidas en relación con variables como superficie-población-actividad económica desarrollada. En lo relativo a los usos de las infraestructuras se pone un especial énfasis en una trilogía de carácter económico, social y empresarial, con una valoración crítica sobre la gestión pública- 
privada-concertada en algunas infraestructuras (lacono, M., Levinson, D., y ElGeneidy, A., 2008).

La estructura de una gran parte de dichas redes regionales se basa - como en cualquier otro lugar del mundo - en hubs destacados y redes pretéritas, con ciertas posibilidades de "hubing" (creación de nuevos hubs) tanto en el transporte por carretera como en el ferroviario de cercanías. La funcionalidad de dichas redes de transporte está sujeta a una serie de interrogantes técnicos y de diseño, entre ellos los siguientes: ¿cabe la participación de las redes regionales en sistemas de red con carácter dominante?, ¿pueden ensayarse y llevarse a la práctica sistemas hub and spoke a escala regional?, ¿cuál es el futuro de las mini-redes?, y por último ¿es posible una reformulación de las redes regionales?, aunque la pregunta final es si resueltos los interrogantes mencionados, es realmente posible un diseño a escala regional compatible y complementario con las grandes actuaciones de dimensión estatal o supracomunitarios.

\section{REDES DE TRANSPORTE Y LOGÍSTICA INTEGRADA: ALGUNOS ESTUDIOS DE CASO.}

Las redes de transporte son indisolubles de los programas logísticos que las soportan. La aparición de los primeros "Computer Reservation Systems" (CRS), se debe precisamente a las necesidades de información y gestión del sector del transporte aéreo y del sector turístico, dado el enorme caudal de información necesaria para el buen desarrollo de las actividades comerciales aéreas y del propio desenvolvimiento del turismo de masas, en cuanto a reservas, transportes, hoteles y demás servicios vinculados. Caso significativo es el de Amadeus, creado por cuatro compañías aéreas europeas, para poder realizar eficazmente su sistema de reservas aéreas, ventas y demás servicios generales y turísticos asociados (Cuadro 1).

Más tarde, el paulatino incremento en el volumen del tráfico aéreo y del turismo en general ha propiciado que se constituyan los "Global Distribution Systems" (GDS), en orden a atender y dar servicio a las crecientes demandas de información y distribución da datos en el campo de los transportes y el turismo internacional.

Un estudio pormenorizado de Amadeus, como caso paradigmático, permite observar la variedad de servicios que oferta, así como la paulatina expansión a otras áreas de negocios próximas, tales como la gestión de websites, agencias de viajes y reservas de hoteles, alquiler de coches o tiendas virtuales:

Amadeus es la compañía de referencia mundial en el procesamiento de transacciones y proveedor de soluciones tecnológicas avanzadas para el sector mundial del viaje y el turismo. Sus ratios operativas, con cifras de 2011, son espectaculares: 
Cuadro 1. Grandes Computer Reservations Systems del mundo.

\begin{tabular}{|l|c|c|c|}
\hline \multicolumn{1}{|c|}{ NOMBRE } & CREADORES & $\begin{array}{c}\text { USADO TAMBIÉN } \\
\text { POR (2010) }\end{array}$ & $\begin{array}{c}\text { \% USA SHARE } \\
\text { (2010) }\end{array}$ \\
\hline AMADEUS & 4 & 7 & 49,2 \\
\hline SABRE & 7 & 13 & 44,7 \\
\hline GALILEO & 10 & 4 & 19,7 \\
\hline WORLSPAN & 3 & 6 & 26,5 \\
\hline TRAVELSKY & 4 & 4 & \\
\hline PATHEO & 3 & 5 & \\
\hline ABACUS & 12 & 4 & \\
\hline KIU & 13 & 3 & \\
\hline SHARES & 6 & & \\
\hline
\end{tabular}

Fuente: Elaboración propia con datos de la WTO, 2010.

$-115,9 \mathrm{mll} /$ pax embarcados

- Proveedor de servicios para 43 compañías aéreas

- La plataforma de reservas Altéa alcanza a 120 compañías aéreas

- 948 mll. de transacciones de viaje

Sus ratios económicas son, con cifras de 2011, igualmente destacadas:

- Beneficios netos $2707 \mathrm{mll} / €$

- Las reservas crecieron un 6,1 \%, (132 mll.)

- Las reservas a través de agencias de viajes crecieron 6,7 \% (115,9 mll)

- Incremento de pasajeros embarcados 23,3 \% (21,9 mll más)

- Los Ingresos crecieron un $8 \%$, por incremento de la cuota mundial de $0,9=$ a nivel mundial $38,2 \%$

Sus ratios relativas a redes, con cifras de 2011 , son de las mayores a nivel mundial:

- Sedes en Madrid (oficinas centrales), Niza (desarrollo) y Erding (operaciones, centro de procesamiento de datos)

- Delegaciones regionales en Miami, Buenos Aires, Bangkok y Dubái.

- Mantiene relaciones con clientes a través de 73 organizaciones

ISSN: 0212-8594 ISSN-e: 2340-2776. № DOI:http://dx.doi.org/10.12795/rea.2013.i30.02 


\author{
comerciales (ACO, Amadeus Commercial Organisations), que cubren \\ 195 países \\ - Empleo: 10.000 trabajadores, de 123 nacionalidades
}

La mera enumeración de las cifras de actividad de Amadeus pone en los antecedentes de su valor económico y fáctico a nivel internacional, tanto como de la imprescindible función que desempeña facilitando labores generales y particulares de logística aplicada a las compañías que tienen contratados sus servicios.

La logística global moderna vehicula el vector que va desde el fabricante-productor al distribuidor-mayorista-intermediario, y finalmente al consumidor, encargándose de asegurar una óptima relación y disponibilidad de recursos cara al proceso del transporte. La logística global comprende diversos tipos de valor agregado a través de la rentabilidad del sistema y de la generación de capital, de modo que se produce una incidencia positiva en las ventas, limitando los costes de operación, aportando la renta derivada del capital-trabajo y favoreciendo las inversiones (Windt, K. y Huismann, M., 2007).

El comercio y la distribución de la carga se presentan como un factor de globalización, en tanto que la logística global se constituye como una nueva estrategia corporativa. Los atributos logísticos más sobresalientes dentro de esta concepción se centran en la disminución de costes, empleo continuado de sistemas globales, utilización del transporte multimodal como canal preferente de distribución, contempla el empleo de sistemas y flujos de información sin demoras ni distorsiones y la propiedad compartida de la información y la visibilidad.

Tres ejemplos de caso significativos sirven para desarrollar lo enunciado en el párrafo precedente: el centro de distribución mundial de FEDEX en Memphis, el sistema de distribución de la empresa alimentaria Bimbo y los servicios de Suply Nexus en el campo del "multichannel retail".

FEDEX EXPRESS es la mayor empresa de transporte del mundo, cuyo centro de distribución mundial se encuentra en Menphis. Según el Institute of Transport Management, su hub de Menphis puede ser considerado como el mejor centro de carga global en 2011, contando con grandes capacidades de transporte en el hinterland de la ciudad de Menphis, tanto por carretera como por ferrocarril, transporte fluvial o aéreo. Desarrolla flujos de entrega vespertinos, transporte nocturno y distribución al día siguiente en cualquier lugar del mundo, lo que justifica su destacado liderazgo global. Además, su hub de Menphis ofrece una alta conectividad entre Norteamérica y Asia, utilizando como flota preferente la aeronave B-777-F específica para carga, disminuyendo así sus costes operativos en cuanto a adquisición y mantenimiento de flota. Otros de sus atributos más conocidos es su compromiso por la excelencia, por lo que se somete a continuadas metaevaluaciones para mantener su estándar de calidad en los servicios que ofrece. 
Un segundo ejemplo emblemático es el que procede del sistema de distribución de productos alimentarios BIMBO. Tres son los vectores de actuación elegidos por dicha multinacional, en los que el papel del transporte se muestra determinante. Desde el proveedor se crea un flujo de materiales y productos en cantidad, calidad y oportunidad, transportado por una red propia de carácter mayorista acompañada de asociaciones con múltiples distribuidores minoristas consorciados, asegurando la llegada a mercado de dicha producción. Los clientes minoristas de la multinacional reportan sus pedidos en línea, éstos son preparados 33 horas antes de ser entregados al cliente y con ello se asegura del mismo modo un caudal de efectivo monetario, empleado para el proceso productivo de fabricación, aportando liquidez real y permitiendo el almacenamiento y despacho de la producción, constituyendo de ese modo un círculo virtuoso en el que la rapidez en el transporte y producción adquieren un carácter estratégico para la empresa.

El tercer ejemplo a colación se refiere a la distribución multicanal y "suply chain" de la compañía SUPPLY NEXUS, entendiendo por supply chain - más allá de la traducción literal del inglés como "cadena de abastecimiento"- un sistema de organizaciones, personal, tecnología, información y actividades implicadas en la movilidad de productos y servicios directamente del productor al consumidor. Se trata en este caso de una empresa de carácter global especializada en el diseño e implementación de soluciones de distribución multicanal y supply chain, empleando tecnología de Manhattan Associates, líder mundial en software y soluciones en supply chain, compitiendo mediante tecnologías lideres, mejores prácticas y recursos experimentados, lo que les permite ofertar soluciones avanzadas (Gokhan, N. y Needy, N., 2010).

Esta empresa según su propia definición, está comprometida con la búsqueda continua de la excelencia operativa a través de la innovación y aplicaciones de nuevas tecnologías en los campos de la distribución y del "supply chain management" (Ehmre, J., Meisel, S. y Engelman, S., 2009), entendido como la eficiencia de la cadena de suministro o el abastecimiento por el proveedor a clientes. Su definición de la cadena de suministro-gestión se fundamenta en que el supply chain management abarca la planificación y gestión de todas las actividades involucradas en la conversión de recursos y abastecimiento, así como todas las actividades de gestión de la logística. En lo que toca a los transportes su planteamiento reside en tres criterios: velocidad, productividad y precisión-exactitud, criterios practicados en un hub en el que se realizan labores de general management, optimización de slots (frecuencias de distribución en este caso) y management administrativo específico, atendiendo las diversas escalas en la demanda y dando un cuidado servicio al cliente. 


\section{REDES DE TRANSPORTE Y ARTICULACIÓN TERRITORIAL.}

Las redes de transporte presentan un indudable protagonismo en la articulación del territorio, y en ese caso las comunicaciones y telecomunicaciones vinculadas al transporte se apoyan en los sistemas y redes de comunicación como factor estratégico y operativo en el mundo de la globalización. En todo ello incide la presencia de comunicaciones fijas y a distancia (redes fijas), las comunicaciones con soporte inalámbrico, las nuevas tecnologías basadas en el uso del satélite y los ductos, conformándose en su conjunto como un grupo de factores condicionantes de las accesibilidades o desenclaves, y especialmente como factor determinante del comercio.

La internacionalización en los transportes se asocia con procedimientos de difusión de procesos, transferencias, intercambio de información, etc., que acompañan en su desarrollo operativo a los flujos de pasajeros y mercancías. Va a ser precisamente con los "spillovers" generados, con los que se cree un valor añadido que se transfiere al entorno como consecuencia de su actuación e influencia sobre los sistemas productivos.

Como consecuencia, el concepto tradicional de espacio se transforma como resultado del desarrollo de las redes de transporte, advirtiéndose varios grupos de factores determinantes en tal proceso: las infraestructuras de transporte se convierten en factor de atracción de industrias y servicios, al tiempo que se generan modelos precisos de localización espacial atendiendo a la ubicación de nodos, hubs y mallas de transporte. Las políticas de organización y distribución de infraestructuras de transporte ocasionan diferentes impactos socioeconómicos y territoriales derivados de la toma de decisiones. Por último, se establece una relación dialéctica a través del papel que desempeñan los actores que intervienen en el diseño territorial (grandes actores y actores locales), sin olvidar la labor de lobbie que ejercen las grandes cadenas logísticas a la hora de fijar sus emplazamientos.

Otro tema de especial interés en lo relativo a las aportaciones de la red de transportes a la articulación territorial, es el que se centra en el debate sobre la accesibilidad sostenible, entendido como la necesidad de una movilidad adecuada a las exigencias económicas y sociales del momento, dependiendo del nivel de desarrollo societario y de sus capacidades para introducir sinergias creativas (Axhaunsen, K., 2007). El papel de las políticas en la accesibilidad sostenible muestra en general un discurso cambiante y acomodado a directrices coyunturales, con disimetrías de análisis y aplicación de criterios según entornos determinados, dentro de los cuales se puede contemplar incluyo la involución territorial (Anas, A. y Liu, Y., 2007). En cuanto a los impactos relativos a la problemática de la accesibilidad sostenible, se concentra la preocupación por los espacios con accesibilidad deficiente, los fenómenos de concentración o

ISSN: 0212-8594 ISSN-e: 2340-2776. № DOI:http://dx.doi.org/10.12795/rea.2013.i30.02 
dispersión de infraestructuras, los de congestión y problemática de la contaminación vehicular o a la asociada a grandes centros de transporte.

No se atisban soluciones generales plausibles para casos concretos, pero si se perciben bajo la forma de retos, como el de la eficiencia de los sistemas de transporte en sus facetas de transporte urbano, regional o internacional; el del consumo energético más acentuado si cabe en períodos de crisis; la definición de las políticas de actuación en el campo de las redes de transporte; las disimetrías existentes en los diversos grados de desarrollo territorial en el mundo, y las disimetrías propias dentro de la misma escala regional según qué lugar del mundo se trate (Mundo desarrollado, Unión Europea en particular, Asia desarrollada, países emergentes, planes nacionales, planes regionales y locales).

\section{BIBLIOGRAFÍA}

Adey, P. (2009): Mobility. Key ideas in geography. Nueva York-Londres, Routledge.

Anas, A. y Liu, Y. (2007): "A regional economy, land use and transportation model (RELUTRANS): formulation, algorithm design and testing". Journal of Regional Science, 47, 3, 415455. http://dx.doi.org/10.1111/j.1467-9787.2007.00515.x

Axhaunsen, K. (2007): Accesibilities: long-term perspectives. International Conference "access to destination". Minneapolis, University of Minnesota,

Baker, J. y Macdonald, M. (2006): Sustainable transport choices and the retail sector. Londres, Planning for sustainable land use and transport, European Transport Conference.

Cresswell, T. (2006): On the move: mobility in the modern western world. Londres, Routledge.

Dandrea, A.; Ciolfi, I. y Gray, B. (2011): "Methodological challenges and innovations in mobilities Mobilities, 6, http://dx.doi.org/10.1080/17450101.2011.552769

Dangelmaier, M. et al. (2011): "Generation of scenarios, preliminary assessment of safety effects and initial prioritization to increase the self-explaing and forgiving character of road environments". BEKIARIS, E.-WIETHOFF,M (Eds.). En Infrastructure and safety in a collaborative world, Nueva York, Springer.

De Brucker, K.; Macharis, C. y Verbeke, A. (2011): "Multicriteria analysis in transport project evaluation: an institutional approach". European Transport, 47, 3-24.

De Dios Ortuzar, J. y Willumsen, G. (2001): Modelling transport. Nueva York, Wiley and Sons.

Dooms, M. et al. (2007b): "An application of stakeholder analysis to infrastructure development: the case of the DHL super-hub location choice. En Transport project evaluation.

ISSN: 0212-8594 ISSN-e: 2340-2776. № DOI:http://dx.doi.org/10.12795/rea.2013.i30.02

REA 30 (2013):27-47

http://www.publius.us.es/estudios_andaluces 
Extending the social cost-benefits approach", HAEZENDONCK, E. (Ed.). Chetelham, Edward Elgar.

Dooms, M. (2010): Crafting the integrative value proposition for large scale transport infrastructure hubs: a stakeholder management approach. Bruselas, VUB Press.

Ehmre, J.; Meisel, S. y Engelman, S. (2009): "Data chain management for planning in city logistics". International Journal of Data Mining, Modelling and Management, 1, 4, 335-356. http://dx.doi.org/10.1504/IJDMMM.2009.029030

Elhedeli, S. y Hu, F. (2005): "Hub-and-spoke network design with congestion". Computers \& Operation research, 32, 1615-1632.

European Transport Conference (2006): Sustainable transport choices and the retail sector. Londres, Planning for sustainable land use and transport, European Transport Conference.

Garrison, W. y Levinson, D. (2006): The transportation experience: policy, planning and deployment. Oxford, Oxford University Press.

Gokhan, N. y Needy, N. (2010) "Development of a simultaneous design for supply chain process for the optimization of the product design and supply chain configuration problem". Engineering Management Journal, 22, 4, 20-30.

Guneri, A.; Cengiz, M. y Seker, S. (2009): "A fuzzy ANP approach to shipyard location selection". Expert Systems with Application, 36, 4, 7992-7999. http://dx.doi.org/10.1016/j.eswa.2008.10.059

Haezendonck, E. (2007): Transport project evaluation. Extending the social cost-benefits approach. Chetelham, Edward Elgar.

Hakimian, H. y Zandi, M. (2009): Foliated transportation Systems. Boras, Universidad de Boras.

Haynes, P. (2010): "Information and communication technology and international business travel: mobilities allies ?". Mobile Computing, Mobilities y Journal of Urban Technology (Edición especial virtual).

Hensher, D. (2004): Handbook of transport geography and spatial systems. Bingley, Emerald Group Publishing

Hensher, D. y Puckett, S. (2005): "Refocusing the modlleing of freight distribution: development of an economic-based framework to evaluate supply chain behaviour in response to congestion charging". Transportation, 32, 573-602. http://dx.doi.org/10.1007/s11116-0047615-6

Hilletoft, P. (2009): "How to develop a differenciated supply chain strategy". Industrial Management \& Data Systems, 109, 1, 16-33. http://dx.doi.org/10.1108/02635570910926573

ISSN: 0212-8594 ISSN-e: 2340-2776. № DOI:http://dx.doi.org/10.12795/rea.2013.i30.02 REA 30 (2013):27-47

http://www.publius.us.es/estudios_andaluces 
lacono, M., Levinson, D. y El-Geneidy, A. (2008): "Models of transportation and land use change. A guide to the territory". Journal of Planning Literature, 22, 323-340. http://dx.doi.org/10.1177/0885412207314010

International Transport Forum (2008): Transport and energy. The challenge of climate change. París, International Transport Forum.

Ison, S. et al. (2011): "UK regional airport commercialisation and privatisation: 25 years on". Journal of Transport Geography, vol. 19, 1341-1349. http://dx.doi.org/10.1016/j.jtrangeo.2011.06.005

JENSEN, A. (2011): "Mobility, space and power: on the multiplicities of seeing mobility". Mobilities, vol. 6, 2, 255-271. http://dx.doi.org/10.1080/17450101.2011.552903

Kile, J. (2011): The highway trust fund and paying for highways. Washington, Congressional Dudget Office.

Kalantari, J. y Lumdsen, K. (2007): Stepwise replacement of direct shipment network wit a hub and spoke system. Reykjavik, NOFOMA.

Kalantari, J. y Sternberg, H. (2009): "Research outlook on a mixed model transportation network". European Transport, 41, 1-18.

Knockaert, J.; Evangelinos. C.; Rietveld, P y Wieland, B. (2009): “Differentiated infrastructure charging: a comparison on theory and practise". European Transport, 43, 4-34.

Knowles, R.; Shaw, J. y Docherty, I. (2008): Transport geographies: mobilities, flows and spaces. Chichester, Blackwell.

Koh, A.; Sheperd, S. y Sumalle, S. (2009): "Second best toll and capacity optimisation in netwoks: solution algorithm and policy implications". Transportation, 36, 2, 147-165. http://dx.doi.org/10.1007/s11116-009-9187-y

Levinson, D. (2008): "Density and dispersion: the codevelopment of land use and rail in London". Journal of Economic Geography, 8, 1, pp. 55-77.

http://dx.doi.org/10.1093/jeg/lbm038

Levinson, D. (2011): "The evolution of transport and land use: an introduction to the special issue and an outline of a research agenda". Journal of Transport and Land Use, vol. 4, 2, 1-3. http://dx.doi.org/10.5198/jtlu.v4i2.332

Lewis, E. (2006): Future models of retail logistics in age of e-comerce. Londres, Planning for sustainable land use and transport, European Transport Conference.

Liu, J. y Chan, C. (2003): "Mixed truck delivery systems with both hub-and-spoke and direct shipment". Transportation Research Part E, 39, 325-339. http://dx.doi.org/10.1016/S13665545(03)00005-X

ISSN: 0212-8594 ISSN-e: 2340-2776. № DOI:http://dx.doi.org/10.12795/rea.2013.i30.02 
Mackinnon, A. (2006): "A review of European truck tolling schemes and assessment of their possible impact on logistics systems". International Journal of Logistics: Research and Applications, 9, 3, 191-205. http://dx.doi.org/10.1080/13675560600859110

Mackinnon, D.; Shaw, J. y Docherty, I (2008): Diverging mobilities: devolution, transport and policy innovation. Bingley, Emerald Group Publishing.

Mcdonnald, M. (2006): Intelligent transport systems In Europe: oportunities for future research. Londres, World Scientific.

Marcucci, E y Danielis, R. (2008): "The potential demand for an urban freight consolidation centre". Transportation, vol. 35, pp. 269-284. http://dx.doi.org/10.1007/s11116-007-9147-3

Mees, P. (2010): Transport for suburbia: beyond the automobile age. Londres, Earthscan.

Meunier, D. y Quinet, E. (2009): "Effect of imperfect competition on infrastructure charges". European Transport, 43, 113-136.

Nagurney, A. (2006): Supply chain network economics: dynamics of prices, flows and profits. Cheltenham, Edward Elgar Publishing.

Notteboom, T. (2007): "Concession agreements as port governance tools", in BROOKS, M y CULLINAME, K. (Eds.), Devolution, port governance and performance, pp. 449-467, Elsevier, London.

Okumura, M.; Tsukai, M y Kimura, Y. (2010): "Programation of improvement effects of critical inter-city link. The japanese decadal change of the available travel routes". Journal of Eastern Asia Society for Transportation Studies, Travel demand analysis and forecast, 8, 325-335.

Persson, P. (2006a): Traceability of goods in a transportation network and the potential impact on logistics and transportation services. Izmir, $4^{\text {th }}$ International logistics and supply chain congress.

Persson, P. (2006b): "Identification technologies in transportation: in the context of foliated transportation networks". Australasian Transport Research Forum, vol. 29.

Pitfield, D. (2009): "The assessment of the EU-US open skies agreement: the counterfactual and other difficulties". Journal of Air Transport management, 15, 6, 308-314. http://dx.doi.org/10.1016/j.jairtraman.2009.04.002

Prashler, J. y Bekhor, S. (2004): "Route Choice models used in the stochastic user equilibrium problem: a review". Transportation Review, 24, 4, 437-463. http://dx.doi.org/10.1080/0144164042000181707

Quak, J. y Koster, M (2006): Retailers distribution and local time-window policies, in E. TANIGUCHI Y R. THOMPSON (Eds.). Recent advances in city logistics, Amsterdam, Elsevier.

ISSN: 0212-8594 ISSN-e: 2340-2776. № DOI:http://dx.doi.org/10.12795/rea.2013.i30.02 REA 30 (2013):27-47

http://www.publius.us.es/estudios_andaluces 
Rodriguez, J.P.; Contois, C. y Slack, B (2009): The geography of transport systems. Londres, Routledge.

Roth, G. (2006): Street smart: competition, entrepreneurship and the future of roads. Oakland, The Independent Institut,

Sánchez-Borrás, M. y López, A. (2009): "Rail Infrastructure Charging Systems for High-Speed Lines in Europe". Transportation Research Board, Annual Meeting 2009 Paper no 09-1278.

Shiftan, Y.; Bekhor, S. y Albert, G. (2010): "Route choice behaviour with pre-trip travel time information - a combined rp/sp approach". IET Intelligent Transport Systems, vol. V, issue 3, 183-189.

Southworth, F. (2010): Freight flow models. Intermodal transportation: moving freight in a global economy. Washington, ENO Foundation.

SMall, K. y Verhoef, E. (2007): The economics of urban transportation. Routledge, London.

Srai, J. y Gregory, M. (2008): “A supply network configuration perspective on International supply chain development". International Journal of Operations \& Production Management, $28,5,386.411$.

Stagl, S. (2007): SDRN Rapid research and evidence review on emerging methods for sustainable valuation and appraisal. A report to the sustainable development research network. Londres, Policy Studies Institute.

Susilawati, S., Taylor, M. Y Somenahalli, S. (2010): "Travel time reability measurement for selected corridors in the Adelaide metropolitan area". Journal of the Eastern Asia Society for Transportation Studies, Transportation general, 8, 86-102.

Timilsina, G. y Dulal, H. (2009): "Regulatory instruments to control environmental externalities from the transport sector". European Transport, 41, 80-112.

Uury, P. (2007): Mobilities. Cambridge, Polyty.

Verhoeven, P. (2006): "Port management reform in Europe: is there a role for the EU ?. En Ports are more than piers", in T. NOTTEBOOM (Ed.). Antwerpen, De Lloyd.

Verhoeven, P. (2008): European ports policy: meeting contemporary governance challenges. Dalian, Proceedings of the 2008 IAME Conference.

Wilson, M. (2007): "The impact of transportation disruptions on supply chain performance". Transportation Research Part E, 43, 4, 295-320. http://dx.doi.org/10.1016/j.tre.2005.09.008

Windt, K. y Huismann, M. (2007): “Changing paradigma in logistics. Understanding the shift from conventional control to autonomous cooperation and control", in M. Hilsmann y K, Windt (Eds.), Understanding autonomous cooperation and control in logistics, 1-71.

ISSN: 0212-8594 ISSN-e: 2340-2776. № DOI:http://dx.doi.org/10.12795/rea.2013.i30.02 REA 30 (2013):27-47 
Witlox, F. (2006): "The Iron Rhine railway link: a chronicle of Duth-Flemish geo-politics base on contextual history". The Journal of European Economic History, 35, 1, 149-173

Wu, J.; Sui, Y. y Wang, T. (2009): "Intelligent transport systems in China". Municipal Engineer, 162, 25-32. http://dx.doi.org/10.1680/muen.2009.162.1.25

Xie, F. y Levinson, D. (2007b): "Measuring the structure of road networks". Geographical Analysis, 39, 3, 336-356. http://dx.doi.org/10.1111/j.1538-4632.2007.00707.x

Xie, F. y Levinson, D. (2010): "How streetcars shaped suburbanización: a granger-causality analysis of land use and transit in the Twin Cities". Journal of Economic Geography, 10, 3, pp. 453-470. http://dx.doi.org/10.1093/jeg/lbp031

Wu, J. (2004): Introduction to transport systems. Taipei, Taiwan Press Co. Ltd.

Yang, H.; Feng, X. y Hung, H. (2009): "Private road competition and equilibrium with traffic equilibrium constraints". Journal of Advanced, 43, 1, pp. 23-45.

Yang, Z. y Chen, K. (2010): “Optimization of shipping network of trunk feeder lines for interregional and intra-regional container transport, Asia". Journal of the Eastern Asia Society for Transportation Studies, Logistics and freight transportation, 8, 694-705.

Yerra, B. y Levinson, D. (2005): "The emergency of hierarchy in transportation Networks". Annals of Regional Science, 39, 3, 541-553. http://dx.doi.org/10.1007/s00168-005-0230-4 